\title{
Some Notes on $k$-minimality
}

\author{
Azam Etemad Dehkordy* \\ Department of Mathematical Sciences, Isfahan University of Technology, Isfahan, I- \\ ran.
}

Received April 4, 2017; Accepted September 13, 2018

\begin{abstract}
The concept of minimality is generalized in different ways, one of which is the definition of $k$-minimality. In this paper $k$-minimality is studied for minimal hypersurfaces of a Euclidean space under different conditions on the number of principal curvatures. We will also give a counterexample to $L_{k}$-conjecture.
\end{abstract}

AMS subject classifications: 53D12, 53C40, 53C42.

Key words: $k$-minimal, minimal hypersurface, $L_{k}-$ conjecture.

\section{Introduction}

Let $x: M \rightarrow \mathbb{E}^{m}$ be an isometric immersion from a Riemannian $n$-manifold into a Euclidean space. Denote the Laplacian, the position vector and the mean curvature vector field of $M$, respectively, by $\Delta, x$ and $\vec{H}$. Then, $M$ is called a biharmonic submanifold if $\Delta \vec{H}=0$. Beltrami's formula, $\Delta x=-n \vec{H}$, implies that every minimal submanifold of $\mathbb{E}^{m}$ is a biharmonic submanifold.

Chen initiated the study of biharmonic submanifolds in the mid 1980s [4]. Then, Chen and other authors proved that, in specific cases, a biharmonic submanifold is a minimal submanifold $[4,5,7]$ and Chen introduced his famous conjecture [3]. This conjecture remains open, although the study thereof is active nowadays. Among other results, it is proved in [6] that Chen's Conjecture is true for biharmonic hypersurfaces with three distinct principal curvatures in $\mathbb{E}^{m}$. Furthermore, under a generic condition, Koiso and Urakawa [8] gave affirmative answer to Chen conjecture.

The linearized operator of $(k+1)$-th mean curvature of a hypersurface, i.e. $H_{k+1}$, is the $\mathrm{L}_{\mathrm{k}}$ operator. The $L_{k}$ operator is a natural generalization of Laplace operator for $k=1, \ldots, n[9,10]$. Let $x: M^{n} \rightarrow \mathbb{E}^{n+1}$ be an isometric immersion from a connected orientable Riemannian hypersurface into the Euclidean space $\mathbb{E}^{n+1}$. It is proved that [1]

$$
L_{k} x=(k+1)\left(\begin{array}{c}
n \\
k+1
\end{array}\right) H_{k+1} N
$$

\footnotetext{
*Corresponding author. Email address: ae110mat@cc.iut.ac.ir (A. Etemad)
} 
where $N$ is the unit normal vector field and $k=0, . ., n-1$.The $L_{k}$-conjecture is as follows.

$L_{k}$-Conjecture. Every $L_{k}$-biharmonic hypersurface, namely a Euclidean hypersurface $x$ : $M^{n} \rightarrow \mathbb{E}^{n+1}$ satisfying the condition $L_{k}^{2} x=0$ for some $k=0, \ldots, n-1$, has zero $(k+1)$-th mean curvature.

A manifold with zero $(k+1)$-th mean curvature is called $k$-minimal for $k=0, . ., n-1$. In 2015, Aminian and Kashani [2] proved the $L_{k}$-conjecture for Euclidean hypersurfaces with at most two principal curvatures. They also proved the $L_{k}$-conjecture for $L_{k}$-finite type hypersurfaces.

In this paper, we prove that the $L_{1}$-conjecture is not true for a connected minimal hypersurface of a Eucldean space with arbitrary number of principal curvatures.

\section{Preliminaries}

In this section, we recall some standard definitions and results from Riemannian geometry. Let $n \geq 2$ and suppose $x: M^{n} \rightarrow \mathbb{E}^{n+1}$ is an isometric immersion from an $n$-dimensional connected Riemannian manifold $M^{n}$ into Euclidean space $\mathbb{E}^{n+1}$.

Let $A$ be the shape operator of this immersion and $\lambda_{1}, \ldots, \lambda_{n}$ be the eigenvalues of this self-adjoint operator. The mean curvature of $M$ is given by

$$
n H=\operatorname{trace} A=\lambda_{1} \ldots . \lambda_{n} \text {. }
$$

The $k$-th mean curvature of $M$ is also defined by

$$
\left(\begin{array}{c}
n \\
k
\end{array}\right) H_{k}=s_{k}
$$

where $s_{0}=1$ and $s_{k}=\sum_{1 \leq i_{1}<\cdots<i_{k} \leq n} \lambda_{i_{1}} \ldots . \lambda_{i_{k}}$, for $k=1, \ldots, n$. It is obvious that $H_{1}=H$ and $S=n(n-1) H_{2}$, where $S$ is the scalar curvature of $M$.

The Newton transformations $P_{k}: C^{\infty}\left(T M^{n}\right) \rightarrow C^{\infty}\left(T M^{n}\right)$ are defined inductively by $P_{0}=I$ and

$$
P_{k}=s_{k} I-A \circ P_{k-1}, 1 \leq k \leq n \text {. }
$$

Therefore,

$$
P_{k}=\sum_{i=0}^{k}(-1)^{i} s_{k-i} A^{i}, \quad 1 \leq k \leq n .
$$

Thus the Cayley-Hamilton theorem implies that $P_{n}=0$. It is well known that each $P_{k}$ is a self-adjoint linear operator which commutes with $A$. For $k=0, \ldots, n$, the second 
order linear differential operator $L_{k}: C^{\infty}\left(M^{n}\right) \rightarrow C^{\infty}\left(M^{n}\right)$, as a natural generalization of the Laplace operator on Euclidean hypersurface $M$, is defined by

$$
L_{k} f=\operatorname{trace}\left(P_{k} \circ \nabla^{2} f\right),
$$

where $\nabla^{2} f$ is metrically equivalent to the Hessian of $f$ and is defined by

$$
\left\langle\left(\nabla^{2} f\right) X, Y\right\rangle=\left\langle\nabla_{X}(\nabla f), Y\right\rangle
$$

for all vector fields $X, Y \in C^{\infty}\left(T M^{n}\right)$. Here $\nabla f$ is the gradient vector field of $f$.

When $k=0, L_{0}=\triangle$. In this case, we have also $A \circ P_{0}=A$ and $L_{0}^{2} x=0$, which means, $M^{n}$ is a biharmonic hypersurface.

\section{1-minimality and counterexample}

In this section, we first consider the minimal hypersurfaces of $\mathbb{E}^{n}$ with three distinct principal curvatures.

Theorem 3.1. Let $x: M^{n} \rightarrow \mathbb{E}^{n+1}$ be an isometric immersion from an $n$-dimensional connected Riemannian manifold $M^{n}$ into Euclidean space $\mathbb{E}^{n+1}$. If $M^{n}$ is a minimal hypersurface with three distinct principal curvatures, then $M^{n}$ cannot be 1-minimal.

Proof. Let $\lambda_{1}=\ldots=\lambda_{p}=\alpha, \lambda_{p+1}=\ldots=\lambda_{p+q}=\beta, \lambda_{p+q+1}=\ldots=\lambda_{n}=\gamma$ be principal curvatures of $M^{n}$, for $1 \leq p \leq n-2$ and $1 \leq q \leq n-2$. Therefor we have

$$
\begin{aligned}
s_{2}= & \frac{1}{2} p(p-1) \alpha^{2}+\frac{1}{2} q(q-1) \beta^{2}+\frac{1}{2}(n-(p+q))(n-(p+q)-1) \gamma^{2}+p q \alpha \beta \\
& +(n-(p+q))(p \alpha+q \beta) \gamma .
\end{aligned}
$$

The minimality of $M^{n}$ yields

$$
\gamma=-\frac{(p \alpha+q \beta)}{n-(p+q)}
$$

So we have

$$
s_{2}=-\frac{1}{2(n-(p+q))}(p \alpha+q \beta)^{2}-\frac{1}{2}\left(p \alpha^{2}+q \beta^{2}\right) .
$$

Now if $M^{n}$ is 1-minimal, then (3.1) implies that

$$
(p \alpha+q \beta)^{2}=-(n-(p+q))\left(p \alpha^{2}+q \beta^{2}\right) .
$$

This concludes that $\alpha=\beta=\gamma=0$, which contradicts the assumption.

Theorem 3.1 can be generalized to an arbitrary number of principal curvatures as in the following theorem, 
Theorem 3.2. Let $x: M^{n} \rightarrow \mathbb{E}^{n+1}$ be an isometric immersion from an n-dimensional connected Riemannian manifold $M^{n}$ into Euclidean space $\mathbb{E}^{n+1}$. If $M^{n}$ is a minimal hypersurface with $k$, $k>1$, distinct principal curvatures, then $M^{n}$ cannot be 1-minimal.

Proof. Let $\lambda_{1}=\ldots=\lambda_{p_{1}}=\alpha_{1}, \lambda_{p_{1}+1}=\ldots=\lambda_{p_{1}+p_{2}}=\alpha_{2}, \ldots, \lambda_{\sum_{i=1}^{k-1} p_{i}+1}=\ldots=\lambda_{n}=\alpha_{k}$ be principal curvatures of $M^{n}$, for $1 \leq p_{i} \leq n-(k-1), 1 \leq i<k-1$, and let $p_{k}=n-\sum_{i=1}^{k-1} p_{i}$. Therefor we have

$$
s_{2}=\frac{1}{2} \sum_{i=1}^{k-1} p_{i}\left(p_{i}-1\right) \alpha_{i}^{2}+\frac{1}{2} p_{k}\left(p_{k}-1\right) \alpha_{k}^{2}+\sum_{1 \leq i \leq j \leq k-1} p_{i} p_{j} \alpha_{i} \alpha_{j}+p_{k}\left(\sum_{i=1}^{k-1} p_{i} \alpha_{i}\right) \alpha_{k} .
$$

We obtain from minimality of $M^{n}$,

$$
\alpha_{k}=-\frac{\sum_{i=1}^{k-1} p_{i} \alpha_{i}}{p_{k}}
$$

so we have

$$
s_{2}=\frac{-1}{2 p_{k}}\left(\sum_{i=1}^{k-1} p_{i} \alpha_{i}\right)^{2}-\frac{1}{2}\left(\sum_{i=1}^{k-1} p_{i} \alpha_{i}^{2}\right) .
$$

Now if $M^{n}$ is 1-minimal, then (3.2) implies that

$$
\left(\sum_{i=1}^{k-1} p_{i} \alpha_{i}\right)^{2}=-p_{k} \sum_{i=1}^{k-1} p_{i} \alpha_{i}^{2}
$$

This concludes that $\alpha_{i}=0$, for $i=1, \ldots, k$, which contradicts the assumption.

An immediate corollary for this section is stated as follows.

Corollary 3.1. Let $x: M^{n} \rightarrow \mathbb{E}^{n+1}$ be an isometric immersion from an $n$-dimensional connected Riemannian manifold $M^{n}$ into Euclidean space $\mathbb{E}^{n+1}$.If $M^{n}$ is a minimal hypersurface, then $M^{n}$ is 1-minimal if and only if $M^{n}$ has exactly one vanishing principal curvature.

We conclude this section with a Counterexample for $L_{k}-$ Conjecture.

Counterexample. By Corollary 3.1, a connected minimal $L_{1}$-biharmonic hypersurface of $\mathbb{E}^{n+1}$ with at least one non zero principal curvature cannot be 1-minimal.

\section{2-minimality}

In this section we study the property of 2-minimality for some hypersurfaces of Euclidean spaces in the specific cases. 
Theorem 4.1. Let $n$ be odd and $x: M^{n} \rightarrow \mathbb{E}^{n+1}$ be an isometric immersion from an $n$-dimensional connected Riemannian manifold $M^{n}$ into Euclidean space $\mathbb{E}^{n+1}$. If $M^{n}$ is a minimal hypersurface with two distinct principal curvatures, then $M^{n}$ cannot be 2-minimal.

Proof. According to the calculation on the page 4 of [2], we have

$$
s_{3}=\sum_{i=0}^{n}\left(\begin{array}{c}
m \\
i
\end{array}\right)\left(\begin{array}{c}
n-m \\
3-i
\end{array}\right) \alpha^{i} \beta^{3-i}
$$

From the minimality of $M^{n}$, it follows that $\alpha=-\frac{n-m}{m} \beta$. Therefore

$$
s_{3}=\sum_{i=0}^{n}\left(\begin{array}{c}
m \\
i
\end{array}\right)\left(\begin{array}{c}
n-m \\
3-i
\end{array}\right)(-1)^{i}\left(\frac{n-m}{m}\right)^{i} \beta^{3} .
$$

Now from (4.1), if $M^{n}$ is 2-minimal, then $\alpha=\beta=0$, which yields a contradiction.

In Theorem 4.1, if $n$ is even, then we have another theorem.

Theorem 4.2. Let $n$ be even and $x: M^{n} \rightarrow \mathbb{E}^{n+1}$ be an isometric immersion from an $n$-dimensional connected Riemannian manifold $M^{n}$ into Euclidean space $\mathbb{E}^{n+1}$. Assume also that $M^{n}$ is a minimal hypersurface with two distinct principal curvatures. If the multiplicity of principal curvatures are equal, then $M^{n}$ is 2-minimal. Otherwise, $M^{n}$ cannot be 2-minimal.

Proof. We have

$$
\sum_{i=0}^{n}\left(\begin{array}{c}
m \\
i
\end{array}\right)\left(\begin{array}{c}
m \\
3-i
\end{array}\right)(-1)^{i}=0
$$

thus if the multiplicity of two principal curvatures are equal, then $s_{3}=0$, by (4.1). This means that, independent of $\beta, M^{n}$ is 2-minimal. In other cases, the proof is similar to the proof of Theorem 4.1.

For three distinct principal curvatures in a spacial case, we have the following theorem.

Theorem 4.3. Let $n \geq 5$ and $x: M^{n} \rightarrow \mathbb{E}^{n+1}$ be an isometric immersion from an $n$-dimensional connected Riemannian manifold $M^{n}$ into Euclidean space $\mathbb{E}^{n+1}$. If $M^{n}$ is a minimal hypersurface with three distinct principal curvatures of multiplicity $\{n-2,1,1\}$, then $M^{n}$ is 2-minimal if and only if the principal curvature of multiplicity $n-2$ is vanish.

Proof. Let $\alpha, \beta, \gamma$ be principal curvatures of $M^{n}$ with multiplicity $n-2,1,1$ respectively. According to definition, we have

$$
s_{3}=\frac{(n-2) !}{3 !(n-5) !} \alpha^{3}+\frac{(n-2)(n-3)}{2} \alpha^{2}(\beta+\gamma)+(n-2) \alpha \beta \gamma .
$$


Since $M^{n}$ is minimal we have $\alpha=-\frac{\beta+\gamma}{n-2}$. So by substitution in (4.2) we get,

$$
s_{3}=T(\beta+\gamma)^{3}-(\beta+\gamma) \beta \gamma=(\beta+\gamma)\left(T \beta^{2}+T \gamma^{2}+(2 T-1) \beta \gamma\right),
$$

where $T=\frac{n^{2}-4 n+3}{3(n-2)^{2}}$. Because of non-vanishing of the second parenthesis in (4.3) for $n \geq 5$, if $M^{n}$ is 2-minimal, we conclude that $\beta=-\gamma$ and $\alpha=0$. The converse is obvious from (4.2).

A direct computation shows that the result of Theorem 4.3 is also true for $n=3$ and $n=4$.

The last theorem is about 2-minimality property for minimal hypersurfaces with three distinct principal curvatures, when the dimension of hypersurface is a multiple of three.

Theorem 4.4. Let $n=3 m$, for some $m \geq 3$ and $x: M^{n} \rightarrow \mathbb{E}^{n+1}$ be an isometric immersion from an $n$-dimensional connected Riemannian manifold $M^{n}$ into Euclidean space $\mathbb{E}^{n+1}$. Assume also that $M^{n}$ is a minimal hypersurface with three principal curvatures of equal multiplicity. Then $M^{n}$ is 2-minimal if at least one of the principal curvatures is zero.

Proof. Let $\alpha, \beta, \gamma$ be principal curvatures of $M^{n}$. By definition of $s_{3}$, we have,

$$
s_{3}=\left(\begin{array}{c}
m \\
3
\end{array}\right)\left(\alpha^{3}+\beta^{3}+\gamma^{3}\right)+m\left(\begin{array}{c}
m \\
2
\end{array}\right)\left(\alpha^{2} \beta+\alpha \beta^{2}+\alpha^{2} \gamma+\alpha \gamma^{2}+\beta^{2} \gamma+\beta \gamma^{2}\right)+m^{3} \alpha \beta \gamma .
$$

Because of minimality of $M^{n}$, we have also $\alpha=-(\beta+\gamma)$. Therefore we get

$$
s_{3}=-m(\beta+\gamma) \beta \gamma
$$

It is concluded that if $M^{n}$ is 2-minimal, then one of the principal curvatures is zero.

In Theorem 4.4, for the case $m=2,2$-minimality of $M^{n}$ is a direct result of its minimality.

\section{References}

[1] L. J. Alias and N. Gürbüs, An extension of Takahashi theorem for the linearized operators of higher order mean curvatures, Geom. Dedicat, 121 (2006), 1957-1978.

[2] M. Aminian and S.M.B. Kashani, $L_{k}$-Biharmonic hypersurfaces in Euclidean space, Taiwanese Journal of Mathematics, 19(3) (2015), 113-119.

[3] B. Y. Chen, Some open problems and conjectures on submanifolds of finite type, Soochow J. Math., 17(2) (1991), 169-188.

[4] B. Y. Chen, Total Mean Curvature and Cubmanifolds of Finite Type, Soochow World Scientific New Jersey, 1984.

[5] F. Defever, Hypersurfaces of $\mathbb{E}^{4}$ with harmonic mean curvature vector, Soochow Math. Nachr., 196 (1998), 61-69. 
[6] Y. Fu, Biharmonic hypersurfaces with three distinct principal curvatures in Euclidean space, Tohoku Math J., 67(3) (2015), 465-479.

[7] T. Hasanis and T. Vlachos, Hypersurfaces in $\mathbb{E}^{4}$ with harmonic mean curvature vector field, Math. Nachr., 172 (1995), 145-169.

[8] N. Koiso and H. Urakawa,, submanifolds in a Riemannian manifold, Osaka J. Math., 55 (2018), 325-346.

[9] R. C. Reilly, Variational properties of functions of the mean curvatures for hypersurfaces in space forms, J. Differential Geom., 8 (1973), 465-477.

[10] H. Rosenberg, Hypersurfaces of constant curvature in space forms, Bull Sci. Math., 117 (1993), 211-239. 